\title{
Acoustic Photometry of Biomedical Parameters for Association with Diabetes and Covid-19
}

\author{
Abdulrahman Imad ${ }^{1}$, Noreha Abdul Malik ${ }^{1 *}$, Belal Ahmed Hamida ${ }^{1}{ }$, Gan Hong Seng ${ }^{2}$, \\ Sheroz Khan ${ }^{3}$ \\ ${ }^{l}$ Department of Electrical and Computer Engineering, International Islamic University Malaysia, 53100 Kuala Lumpur, Malaysia. \\ ${ }^{2}$ Department of Data Science, Universiti Malaysia Kelantan, 16100 UMK City Campus Pengkalan Chepa, Kelantan, Malaysia. \\ ${ }^{3}$ Department of Electrical Engineering, Onaizah College of Engineering and Information Technology, Al-Qassim 2053, Saudi Arabia.
}

\begin{abstract}
Because of their mortality rate, diabetes and COVID-19 are serious diseases. Moreover, people with diabetes are at a higher risk of developing COVID-19 complications. This article therefore proposes a single, non-invasive system that can help people with diabetes and COVID-19 to monitor their health parameters by measuring oxygen saturation (SPO2), heart rate, and body temperature. This is in contrast to other pulse oximeters and previous work reported in the literature. A Max30102 sensor, consisting of two light-emitting diodes (LEDs), can serve as a transmission spectrum to enable three synchronous parameter measurements. Hence, the Max30102 sensor facilitates identification of the relationship between COVID-19 and diabetes in a cost-effective manner. Fifty subjects (20 healthy, 20 diabetic, and 10 with COVID-19), aged 1861 years, were recruited to provide data on heart rate, body temperature, and oxygen saturation, measured in a variety of activities and scenarios. The results showed accuracy of $\pm 97 \%$ for heart rate, $\pm 98 \%$ for body temperature, and $\pm 99 \%$ for oxygen saturation with an enhanced time efficiency of 5-7 seconds in contrast to a commercialized pulse oximeter, which took 10-12 seconds. The results were then compared with those of commercially available pulse oximetry (Oxitech Pulse Oximeter) and a thermometer (Medisana Infrared Thermometer). These results revealed that uncontrolled diabetes can be as dangerous as COVID-19 in terms of high resting heart rate and low oxygen saturation.
\end{abstract}

\section{Keywords:}

Max30102 Sensor;

Diabetes;

COVID-19;

Oxygen Saturation;

Heart Rate;

Body Temperature.

\section{Article History:}

Received: $21 \quad$ November 2021

Revised: $\quad 13$ January 2022

Accepted: 22 January 2022

Published: 07 February 2022

\section{1- Introduction}

Coronavirus disease 2019 (COVID-19) is a serious illness. Its spread has been declared a pandemic by the World Health Organisation (WHO), due to its rapid transmission [1]. COVID-19 first appeared in 2019 as cases of fever with unknown aetiology in the city of Wuhan, China. Coronaviruses bear symptoms of respiratory viral infection, corresponding to the conditions of Middle Eastern Respiratory Syndrome (MERS) and severe acute respiratory syndrome (SARS). Both MERS and SARS are members of the same family of viruses. Consequently, they manifest as identical respiratory-related medical conditions. According to estimates [2], COVID-19 has caused over 2.16 million cases and at least 24.681 thousand deaths in Malaysia alone, a country with a population of 32.7 million. Worldwide, COVID-19 has caused around 4.55 million deaths out of 219 million cases [3] and is closely related to diabetes mellitus, a lifelong medical condition that is characterised by elevated glucose levels in the blood, due to abnormal metabolism.

*CONTACT: norehaa@iium.edu.my, abdulrahmanimad12@gmail.com

DOI: http://dx.doi.org/10.28991/esj-2022-SPER-04

(C) 2022 by the authors. Licensee ESJ, Italy. This is an open access article under the terms and conditions of the Creative Commons Attribution (CC-BY) license (https://creativecommons.org/licenses/by/4.0/). 
Specifically, diabetes mellitus (commonly known simply as diabetes) is a disease wherein the body produces insufficient amounts of insulin for the body to be able to convert sugar into energy. Here, it is the pancreas that is responsible for producing insulin enzymes in the body. In economic terms, the global diabetes industry is worth 376 billion USD annually, predicted to rise to 490 billion USD by the year 2030, when the number of diabetes sufferers is estimated to reach approximately 642 million worldwide [4, 5]. In addition, diabetes is a disease that weakens and impairs innate immunity, rendering patients susceptible to severe infections. Innate immunity is the first line of defence against any infection, especially viral infections like COVID-19 [6]. One study in China, sampling more than 174 COVID-19 patients, revealed that diabetes increased the severity of this viral disease. Besides, patients with higher serum levels of pro-inflammatory markers, such as C-reactive protein, appear to be more likely to develop severe pneumonia [7].

There are two main types of diabetes: type 1 and type 2 . In type 1 , the body produces little insulin, due to the loss of insulin-producing $\beta$-cells in the pancreas, thereby leading to hyperglycaemia. Type 1 is most common in children, but symptoms can also develop in adults. In some type 1 cases, the body cannot produce insulin, with the result that the patient is obliged to self-administer or receive daily insulin injections to maintain his or her glucose levels. In contrast, type 2 is the most common form of diabetes overall, and is mainly linked to obesity and physical inactivity in adults. In type 2 , the body produces insulin but does not respond to it. Thus, this is called insulin resistance. To counter this, a healthy lifestyle and physical activity must be maintained. In most cases, diabetics adhere to a dose of medication to keep their glucose levels under control. However, one other type of diabetes is gestational (GDM), consisting of high blood glucose during pregnancy. It is associated with health complications for both the mother and baby, but usually disappears after pregnancy. Nevertheless, both mother and child are left with a higher risk of developing type 2 diabetes later in life [8-10].

Numerous health complications may be caused by diabetes. However, these complications can be prevented by maintaining healthy blood pressure and healthy glucose and cholesterol levels, namely, within the recommended acceptable range. The acceptable fasting glucose range is 4.4-7.2 mmol/L (80-130 mg/dL), and 3.6-6.05 $\mathrm{mmol} / \mathrm{L}(65-$ $110 \mathrm{mg} / \mathrm{dL}$ ) two hours after a meal. This can be ascertained by monitoring blood glucose every day using suitable blood glucose measurement devices [11]. Meanwhile, the cholesterol range for men over the age of 20 is $8.25-11 \mathrm{mmol} / \mathrm{L}$ $(150-200 \mathrm{mg} / \mathrm{dL})$. A glucose test is therefore an essential part of diabetes management, and there are two methods used: invasive and non-invasive. The invasive technique requires regular finger pricks to take a blood sample for testing, while non-invasive techniques include near infrared spectroscopy (NIR), mid-infrared spectroscopy (MID), dual-wavelength spectroscopy, thermal-emission spectroscopy, reverse iontophoresis, and many others [12]. This paper reports a noninvasive system prototype that can help people with diabetes and COVID-19 monitor their health parameters by using a single system to measure oxygen saturation (SPO2), heart rate, and body temperature.

Heart rate and oxygen saturation are important in the case of diabetics, whereas all three parameters are essential when monitoring COVID-19 patients. The system designed in this study achieved a better time efficiency of 5-7 seconds, compared to a commercialised pulse oximeter, which was found to take 10-12 seconds to generate results. The propotype system described in this paper was also proven to be cost-efficient and user-friendly for those seeking to regularly monitor their health. This paper also reports an analysis of the relationship between diabetes severity, heart rate, oxygen saturation, exercise, and diet, ultimately presenting a conclusive summary of the effects of diabetes on COVID-19 severity in the affected subjects. Nevertheless, it should be mentioned here that certain factors can produce inaccurate or false readings of, for example, hypotensive systolic blood pressure, motion, abnormal haemoglobin levels, and light transmission if blocked by black or blue nail polish, or other factors that can lead to similar inaccuracies. Moreover, this system cannot be used for clinical purposes.

Due to the pandemic and accompanying prolonged lockdowns in Malaysia between 2020 and 2021, the main struggles faced in this study involved data collection. Hence, it was extremely difficult to gather data from the 50 subjects sampled from different regions of Malaysia, while at the same time taking all precautions to ensure the safety of the subjects and crew. For instance, all those involved were required to wear face masks and face shields at all times, while subjects needed to be sanitised on arrival, and the equipment sanitised before use. Once data had been gathered, the equipment was then sanitized again for future use with other subjects.

The remainder of this paper is structured as follows: section 2 details various photometrical measurement procedures and techniques in related work; section 3 presents the system flowchart implemented in the research methodology; section 4 presents the results and analysis, and the paper is concluded in section 5 .

\section{2- Literature Review}

\section{2-1-Pulse Oximetry}

This system can be used to measure blood glucose, heart rate, and oxygen saturation, applying a non-invasive technique with the highest possible accuracy. The prototype is non-invasive, meaning that it is pain-free. It is also 
implemented in real time. Specifically, it consists of a near infrared diode $(940 \mathrm{~nm})$ as a transmission spectrum, two light-emitting diodes (LEDs) $(660 \mathrm{~nm}$ ), and an Arduino controller board. A fingertip photo-plethysmography (PPG) signal analysis was conducted. The results obtained were compared to those of a commercial pulse oximeter, showing $\pm 3 \%$ for pulse rate and $\pm 1 \%$ for oxygen saturation error, respectively. Nevertheless, although this technique promises numerous benefits, the prototype is not suitable for clinical purposes, especially not for diabetic patients with high glucose levels, as it introduces more than $15 \%$ error into the corresponding measurements [13]. Figure 1 describes the system adopted in this study.

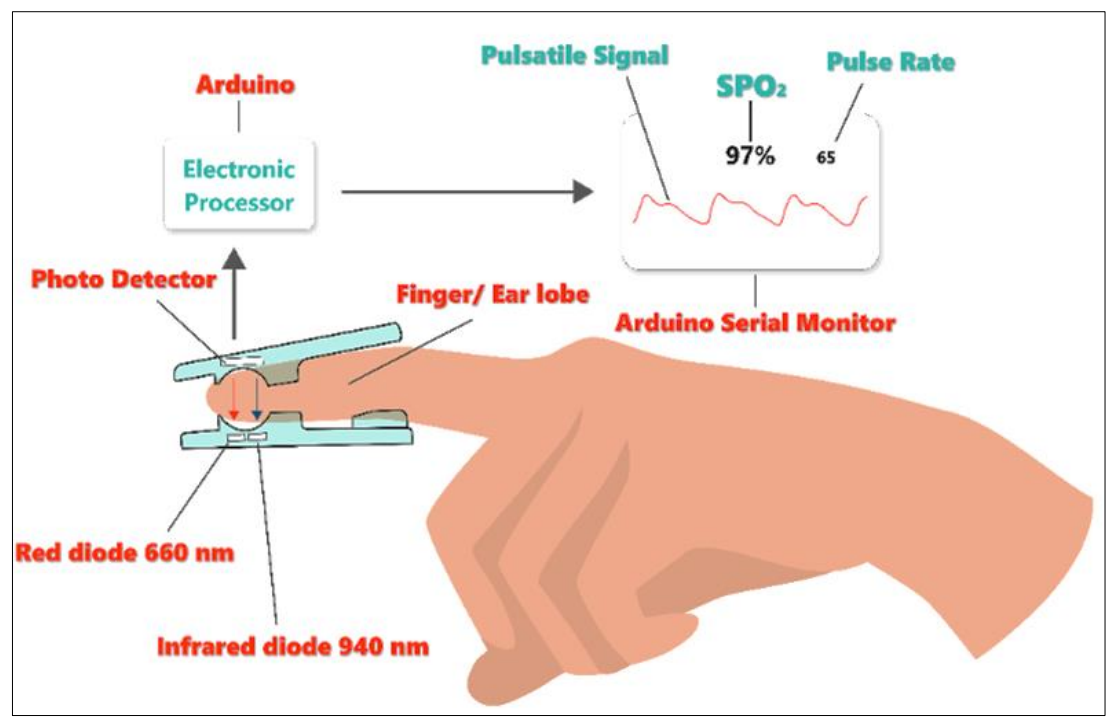

Figure 1. Pulse oximetry system

The purpose of this research is to investigate non-invasive ways of determining blood glucose levels. The procedures involved in pulse oximetry and glucometry rely on light absorption by the body's tissues. Pulse oximetry utlises red (infrared) light with a wavelength of 600-750 nm and infrared light with a wavelength of 850-1000 nm to calculate oxygen saturation in the blood. Glucose is also found in haemoglobin; therefore, the same method can be adopted for blood glucose detection. However, recent studies have found that "Glycemia is a less well-known blood test than conventional blood glucose", despite its significant usefulness, especially where there is suspicion of pre-diabetes or diabetes, or in the case of long-term diabetes monitoring [14].

\section{2-2-Photoacoustic Measurement}

The application of non-invasive blood glucose photometry is demonstrated in [15]. It consists of developing an overview of an experimental setup to generate and collect photoacoustic signals. The above-mentioned test setup is utilised to approve a non-invasive glucose-monitoring technology by gathering photoacoustic estimates from various media simulations. The full range of physiological glucose levels from a $0-500 \mathrm{mg} / \mathrm{dL}$ photometric is observed through changes in glucose concentration. One advantage of adopting this system is that it is not affected by optical dispersion from the body's tissues, unlike conventional optical systems. Any scattered light that is transmitted to the tissues is dissipated into the tissues, creating an acoustic light wave. This technique is also essentially non-destructive, and requires minimal sample preparation prior to estimating glucose levels. Thus, it is safe and easy to use on live tissues for robust long-term glucose monitoring. However, there are also a number of limitations such as long integration time, low signal-to-noise-ratio (SNR), and susceptibility to variations in pulse, temperature, and ambient acoustic noise [16].

In the research reported in [17], a non-invasive glucose-monitoring device was presented, targeting individuals with diabetes via photoacoustic spectroscopy. The sound-shielding features of a box employed in the above-mentioned study were first investigated and evaluated, followed by a photoacoustic cell using an IR-LED and silicon microphone. The frequency properties of natural rubber and sliced ham(s) were then measured with both the sound-shielding box and photoacoustic cells. The experimental findings revealed that the sound-shielding box possessed good sound shielding characteristics to attenuate noise from outside. The correct frequencies were 1.7 to $1.8 \mathrm{kHz}$, which helped modulate the light to achieve a depth of $2-3 \mathrm{~mm}$.

\section{2-3-Hybrid Technique}

In the literature, a novel approach has been proposed for the non-invasive measurement of blood glucose levels [18], using a hybrid technique that combines NIR absorption and bio-impedance measurement. The preliminary results of this hybrid technique have been described. Both techniques were initially tested on an individual basis and then combined 
using least squares regression and an artificial neural network (ANN). The results showed improved accuracy of the combined methods, compared to single methods. This hybrid technique, developed using linear regression models, showed superior results in 10 to $90 \%$ of the data points in regions A and B of the Clarke error grid. These results are considered acceptable. However, the main limitation of this technique is that the model is subjective, which means it can only be used by trained personnel. Moreover, to increase the model's accuracy, it should be capable of obtaining data from different subjects according to a number of criteria such as age, gender, and physical condition. Besides, a temperature sensor should be integrated to calibrate bio-impedance and eliminate the factors affecting NIR readings by adding sensory inputs, such as haemoglobin levels, finger thickness, and colour.

Diabetes mellitus is a chronic disease that is rapidly becoming more and more common across the globe, resulting in millions of diabetics. According to data gathered from the International Diabetes Federation, the estimated number of diabetics worldwide will increase by $10.2 \%$ (578 million) by 2030, and 10.9\% (700 million) by 2045. Machine-learning techniques can and are being used for the early diagnosis of type 2 diabetes to assist patients in adapting to a diet plan and healthier lifestyle habits, thereby avoiding future problems. In this current study, a hybrid strategy was applied to develop an efficient model that could help classify type 2 diabetes. The dataset, obtained from the Biostatistics program at Vanderbilt University in the US and containing information such as age, cholesterol, weight, glucose, and waist/hip ratio among 390 African-American subjects, was relatively imbalanced in terms of diabetic and non-diabetic figures. These features of the dataset were obtained using filter and wrapper methods. Therefore, to balance the dataset, both under- and over-sampling strategies were applied. A voting ensemble model with five algorithms was adopted as a classifier, and the validation model was created using a stratified K-fold technique. Finally, the model's performance was evaluated using recall, F1, precision, and accuracy. When comparing the models with and without sampling, the results showed considerable improvement. The primary goal was to enhance recall while maintaining a reasonable level of precision [19].

\section{2-4-Near-infrared (NIR) Spectroscopy}

The development of a method of NIR spectroscopy for the continuous monitoring of glucose concentration was proposed in [20]. An improved algorithm was consequently presented as a superior and more accurate means of estimating blood-glucose concentration. Polynomial and linear regression analyses were performed to develop this algorithm, using the scattering property of glucose particles and optical imaging principles of PPG. The expected results showed that the predicted glucose concentration following polynomial regression analysis exhibited an error of $+7 \mathrm{mg} / \mathrm{dL}$ while the linear regression exhibited an error of $+12 \mathrm{mg} / \mathrm{dL}$. Thus, it was observed that no linear relationship could be claimed between invasive glucose values and PPG voltage intensity. It was also observed from this study that the polynomial regression method produced better results. However, if the system is implemented, the circuit needs to be integrated with the wireless display and transmitter mode. Moreover, the accuracy of the system could be improved by including a larger number of samples in real time, or by assimilating another machine-learning algorithm that would enable the system for self-learning in future. Furthermore, this system could be improved by integrating it with smartphones for consumers' ease of use.

Diabetes mellitus is a metabolic condition, characterised by persistently elevated blood glucose levels. The blood glucose level of a diabetic must be monitored to control their diabetes. This paper describes the use of a machine-learning technique to predict blood glucose levels based on NIR spectroscopic response data. The NIR spectra for the blood glucose samples were obtained using the NeoSpectraMicro development kit, built in a controlled setting. The experimental dataset was analysed using two machine-learning approaches: first, the random forest algorithm, followed by the support vector machine (SVM) - evaluated as having 67.5\% accuracy - and second, principal component analysis (PCA) and SVM employed in tandem. When compared to the previous technique, PCA preceded by SVM produced a promising outcome with $77.5 \%$ accuracy. The findings imply that NIR spectroscopy, when combined with the appropriate data-modelling method, could be a viable option as a non-invasive system of blood glucose monitoring [21]

\section{2-5-Mid-infrared (MID) Spectroscopy}

Mid-infrared spectroscopy uses light in the spectral range of 2500-10000 nm. Its underlying physical principles are similar to those of NIR, except that MID minimises scattering and maximises absorption because of the broader wavelengths. The spectral band of glucose-generated MID is sharper than the spectral band generated by NIR. However, both NIR and MID have similar limitations: poor and weak penetration due to light characteristics, whereupon MID and NIR only penetrate a few micrometres into the skin. To overcome this limitation, attenuated total reflection (ATR) was employed to enhance light permeation. The MID spectral band for blood glucose is usually applied to the oral mucosa or finger skin, displaying great potential [22]. 
Non-invasive blood glucose monitoring (NGM) refers to technology that is highly challenging for both academic and industrial communities. Therefore, it is desirable to find an appropriate and accurate NGM for patients with hyper- or hypoglycaemia. In comparison with other NGM technologies, infrared is considered to be prospective and the most popular. The testing in this study was conducted with MID spectroscopy, with transmittance varying significantly from $3000 \sim 3500 \mathrm{~cm}^{-1}$ (3333 and $2857 \mathrm{~nm}$ ). As a result, a relationship between MID spectroscopy and blood glucose levels was established. It was possible to use the infrared peak wavelength of $3000 \sim 3500 \mathrm{~cm}^{-1}$ to measure the experiment's physical signals. The intention behind presenting the NGM prototype is to increase motivation for self-management. In NGM research, MID technology holds significant promise and potential [23].

\section{2-6-Iontophoresis}

Iontophoresis has been used for years to transmit medication via the skin by applying an electrical current. Meanwhile, reverse iontophoresis is capable of transporting glucose outward from the skin. This involves the potential application of electricity, with an anode and cathode placed on the skin's surface. This application subsequently causes sodium $\left(\mathrm{Na}^{+}\right)$and chloride $\left(\mathrm{Cl}^{-}\right)$ions from beneath the skin to gravitate towards the cathode/anode, thereby producing an electrical current. In this approach, glucose is measured using an imitative glucose sensor. It is a method that has been utilised in many instances of non-invasive monitoring. However, it is an expensive technique, requiring a long warm-up period of 2-3 hours for adequate glucose levels to become available for measurement [24].

As mentioned earlier, the rate of diabetes occurrence has been increasing annually almost everywhere in the world. Traditional methods of approximating glucose levels are painful, sometimes requiring finger-pricking and thereby posing risk of infection. In contrast, microneedle arrays are painless. However, the integration of a microneedle array with an appropriate electronic device to control medicine delivery has rarely been realised. Thus, in one previous study [25], an iontophoresis-microneedle array patch (IMAP) was developed, powered by a portable smartphone to obtain controlled and active data on the transdermal delivery of insulin. This integrated system was unified into a single patch, allowing for a one-step pharmaceutical administration technique, comprised of "penetration, diffusion, and iontophoresis". The microneedle array in IMAP is pressed against the skin to create microholes, after which the microneedles are withdrawn, and insulin-loaded nanovesicles are delivered via the microholes, using electrically controlled iontophoresis. In a synergistic and highly satisfactory way, this method was found to enhance controlled insulin administration. By adjusting current intensities, IMAP can successfully regulate insulin levels. In the abovementioned in vivo study, IMAP reliably administered insulin and induced significant hypoglycaemia effects in a type 1 diabetic rat model, with superior controllability and efficiency, compared to a pristine microneedle or iontophoresis. The IMAP system therefore implies high potential for diabetes therapy, and the ability to provide both active and longterm glycaemic control without medical assistance [25].

\section{2-7-Thermal Emission Spectroscopy}

Thermal emission spectroscopy methods are grounded in a concept that is similar to the rationale behind clinical membrane thermometers. Here, naturally generated body signals emit infrared signals that can be measured through changes in glucose concentration. The main disparity between this form of glucose monitoring and the application of thermal thermometers is that glucose wavelengths are added, whereupon glucose concentrations in he body can be determined through the fingertip, earlobes, or forearm [26].

Glucose has unique absorptive effects on infrared light, which can be directly linked to its concentration. For example, in the tympanic membrane, glucose-detecting signals from blood vessels need to cross shorter pathways and are therefore suitable for glucose monitoring. This technique has shown good reproducibility for concentrations, but there is strong interference with glucose-detecting signals from body movements and temperature [27].

\section{3- Research Methodology}

The goal in this study was to design a non-invasive system using the Max30102 sensor, in order to monitor and plot heart rate and measure oxygen saturation (SPO2), thereby helping diabetics and COVID-19 patients to maintain a healthy lifestyle. This system would also facilitate the early detection of COVID-19 through estimated SPO2 and heart rate values. Figure 2 presents a flowchart of the system's methodology. 


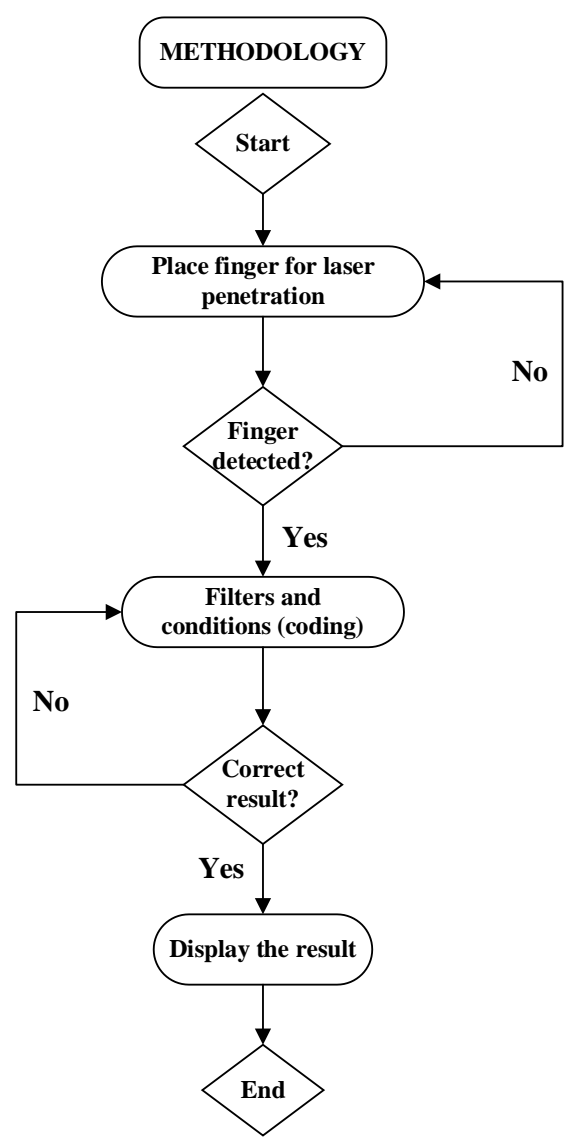

Figure 2. System flowchart, beginning with subjects placing an index finger on the Max30102 sensor. If their index finger is detected, the system will run filters and conditions in Arduino code. If the results match the filters and conditions, they will be displayed on the Oled display screen.

\section{3-1-Experimental Setup}

The experimental setup consisted of the Max30102 sensor, which is a pulse oximeter sensor; an Oled LCD display; a buzzer, and Arduino Uno (a microcontroller board based on the ATmega328P) for the system. Max30102 is composed of low-noise electronics with ambient light rejection capability, internal LEDs, optical elements, and photo-detectors. It functions with $1.8 \mathrm{~V}$ single power supply and another separate power source of $3.3 \mathrm{~V}$ for the internal LEDs. It integrates an infrared and red LED pulse oximeter sensor with an LED pulse to modulate oxygen saturation and estimate heart rate. It was possible to configure the LED current from 0-50 mA with an appropriate supply voltage. For example, the width of the LED display could be configured from $69 \mu$ s to $411 \mu \mathrm{s}$, allowing the system to improve the accuracy of oxygen saturation and heart rate readings. An on-chip temperature sensor can also be integrated into Max30102 to calibrate the temperature dependence of the SPO2 subsystem. Figure 3(a) shows the prototype system, including Arduino. Figure 3(a) also illustrates the system's prototype consists of Arduino Uno controller board, Oled LCD, buzzer and Max30102 sensor. The desired results were obtained by placing an index finger on the LED of the Max30102 sensor, as shown in Figure 3(b).

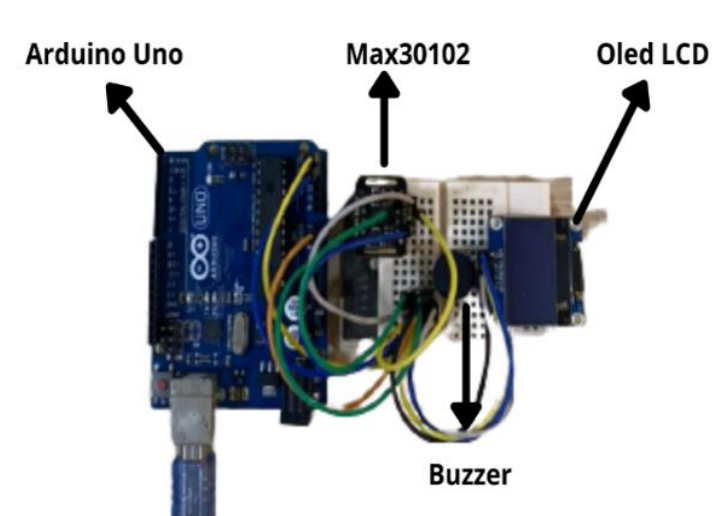

(a)

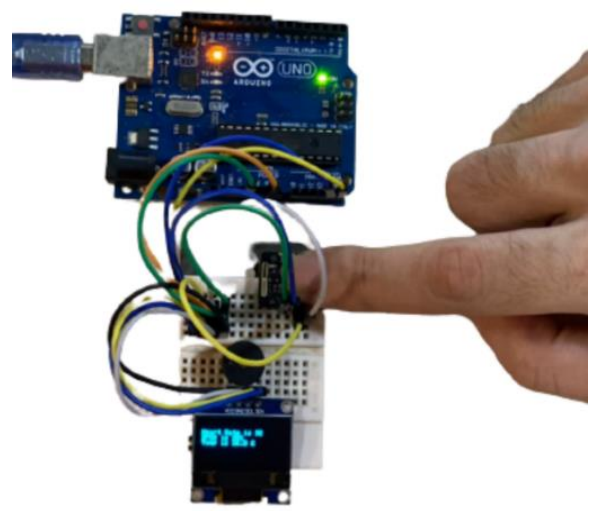

(b)

Figure 3. Prototype of project: (a) labelled prototype, and (b) results obtained through prototype 


\section{3-2- Working Principle of Pulse Oximetry}

A pair of LEDs, addressing a photodiode via a transparent area of the patient's body (in this research, a fingertip), and an electronic processor, were applied in the oximeter. Pulse oximetry deploys two LEDs: one a red LED with a 660 $\mathrm{nm}$ wavelength and the other, an infrared LED with a $940 \mathrm{~nm}$ wavelength. Light absorbance at such wavelengths is clearly different between oxygenated and de-oxygenated blood, or blood that lacks oxygen. Oxygenated blood absorbs higher amounts of infrared light while allowing red light to flow through it, while deoxygenated blood permits infrared light to flow through it while absorbing a greater amount of red light. Figure 4 illustrates the process with oxygenated and deoxygenated haemoglobin.

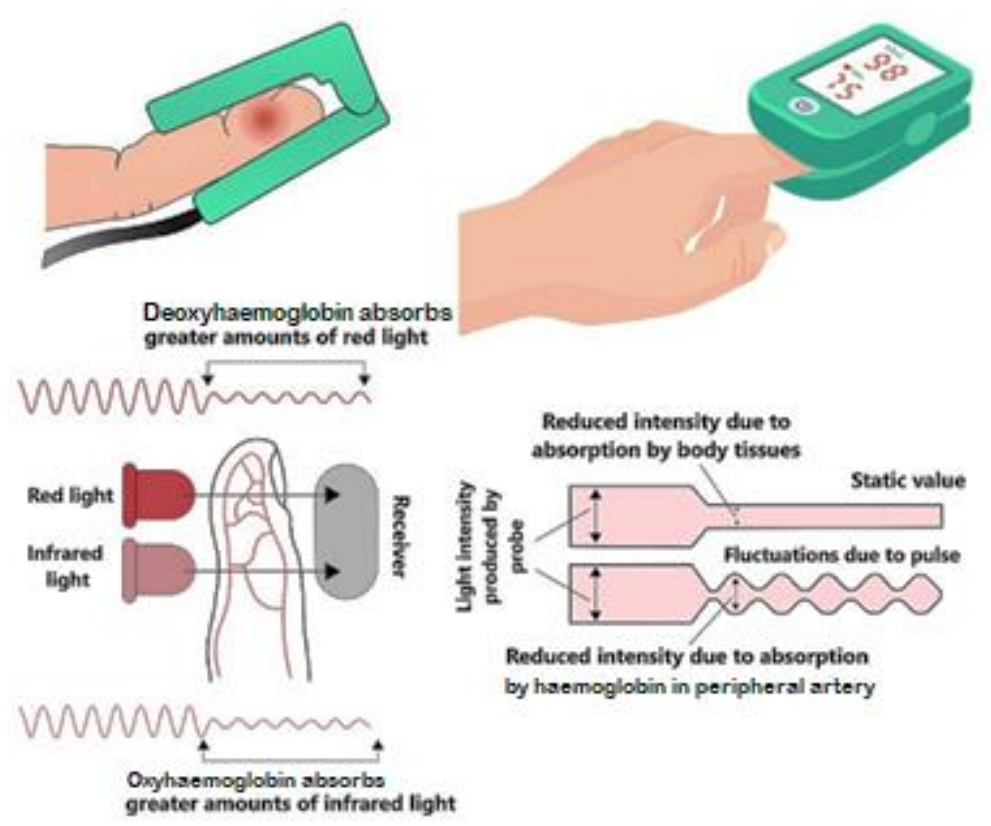

Figure 4. Impact of infrared light on oxygenated and deoxygenated haemoglobin

\section{3-2-1- Oxygen Saturation (SPO2)}

Ambient light cancellation, a discrete-time filter, and a continuous sigma-delta analog-to-digital converter are included in the Max30102 subsystem (ADC). To cancel ambient light and extend the effective dynamic range, an inbuilt track/hold circuit is integrated. The full-scale ranges of the SPO2 ADC are 2-16A. Ambient light cancellation can cancel an ambient current of up to 200A. The inbuilt ADC is a continuous sigma-delta converter with 18-bit resolution for time-oversampling, and a sampling rate of $10.24 \mathrm{MHz}$ with output data ranging from 50-3200 samples per second. The equation used to calculate SPO2 deploys alternating current (AC) and direct current (DC) for the infrared and red-light components, in order to measure SPO2. In addition, it is embedded in the Arduino code.

$$
S P O 2=\frac{A C \text { red } \div D C \text { red }}{A C I R \div D C I R}
$$

\section{3-2-2- Heart Rate}

Blood is pressed into capillaries during each heartbeat, causing the volume of these capillaries to rise gradually. Conversely, their volume drops between heartbeats. This change in volume impacts on the amount of light that can pass through the tissues, such as red or infrared light. Although the fluctuations are small, heart rate can also be approximated by analysing the time-series response to reflected red and infrared light. The Max30102 pulse oximeter is an inexpensive Arduino-compatible sensor, which enables heart rate calculations. Only the red LED is used in the Max30102 heart rate mode to record optical data, thereby finding the user's heart rate and/or PPG.

\section{3-2-3- Temperature}

The Max30102 also includes an attached sensor, which measures temperature in dependence of the oxygen saturation subsystem. The resolution of the sensor is inherent, and at $0.0625^{\circ} \mathrm{C}$. The device's output data are relatively insensitive to the wavelength of the infrared LED, unlike the red LED, and so it is important to correct the interpretation of the data. 


\section{3-3-Data Collection Procedure}

The experiment was carried out on 50 subjects, aged between 18 and 61 years and weighing 60-110 kg. All subjects consented to use of their information for research purposes. Some subjects had conditions such as diabetes and COVID19, while others were healthy (20 diabetic subjects, 10 COVID-19 subjects, and 20 healthy subjects). The subjects rested for five minutes before undertaking the tests, in order to provide a stable reading at rest, except in scenarios that required prior movement. Each subject then placed an index finger on the Max30102 to produce results, which were subsequently compared with those obtained through commercialised pulse oximetry and a thermometer (Oxitech Pulse Oximeter, Medisana Infrared Thermometer, respectively).

\section{4- Results and Analysis}

Results were obtained by plotting the average values derived from the sample of 50 subjects. The standard deviation for heart rate was 12.52. This was a little high, due to the gap value between the various groups. The standard deviation for oxygen saturation was 2.53 , and this was relatively lower, due to the short and low gap values for oxygen saturation. These results were compared to those obtained using commercialised pulse oximeters and other similar work conducted by fellow researchers. In a previous study [13], heart rates and oxygen saturation were measured, with a general accuracy of $\pm 97 \%$ and \pm 99 , respectively, compared to a pulse oximeter in commercial use. In this current study, however, heart rate was measured in different states (unlike [13]), categorised as: resting, exercising, sleeping, and sitting. Moreover, these measurements were taken for different subjects with various health complications or unhealthy habits, such as healthy and unhealthy lifestyles, controlled and uncontrolled diabetes, and COVID-19 and uninfected individuals. The results are detailed below in their respective sections. The advantage of this system was that all three parameters were combined (heart rate, oxygen saturation, temperature) in a single method, unlike [13] and other pulse oximetry approaches in commercial use, where only heart rate and oxygen saturation are measured.

\section{4-1- Heart Rate Result}

Heart rate is an important factor in better understanding individual glycaemic status (in diabetics). There are many factors that can affect heart rate, such as age, activity levels, body position, body size, emotions, medication, cardiovascular disease (CVD), and diabetes. Diabetics tend to have a resting heart rate of over 100 beats per minute (bpm), as opposed to an ideal heart rate of $60-80 \mathrm{bpm}$. Conversely, it can indicate danger if the subject is not a trained athlete but has a resting heart rate of below $60 \mathrm{bpm}$. Researchers have found that hyperglycaemia can cause rapid heartbeat. Studies have likewise shown that major increases in resting heart rate are an indication of type 2 diabetes, while reduced heart rate can indicate the opposite. Furthermore, researchers have discovered that in patients with diabetes, resting heart rate is associated with various types of CVD. Heart rate in any state of rest, deep breathing, recovery, and lying to standing are highest in known diabetic conditions. Resting heart rate can be a crucial factor in the early detection of COVID-19, whereas extreme elevation of an individual's resting heart rate can be an early sign that he or she is infected with COVID-19, or simply requires medical attention [28]. The results in this study were compared to those reported in [13] and for commercialised pulse oximeters, showing a more or less similar accuracy of $97 \%$.

\section{4-1-1- Resting versus Exercising}

Figure 5(a) shows the heart rate results for subjects resting versus exercising, in tests carried out on 30 subjects, excluding COVID-19 patients and subjects with uncontrolled diabetes or other health constraints. Exercise is an important factor in the prevention and control of type 2 diabetes. The ideal resting rate for a healthy individual ranges from $60-80 \mathrm{bpm}$. Heart rate was calculated using the following formula for maximum average bpm:

$$
\text { Maximum average beat per }=220-\text { age }
$$

The age of the sampled subjects is illustrated in Figure 5(a), verifying from 20-32 years. Therefore, the maximum average bpm for the oldest subject was 220-32=188 bpm, and the average heart rate value for exercising was 187 . Therefore, both heart rates fell within the acceptable range, at 78 and 187, respectively.

\section{4-1-2- Sleeping versus Sitting}

Figure 5(b) shows the results for sleeping versus sitting, in tests conducted on 40 of the sampled subjects, excluding COVID-19 patients. The ideal heart rate for a healthy person in a sitting/resting position is $60-80 \mathrm{bpm}$. In contrast, the sleep state usually provides the lowest heartbeat readings. Therefore, both readings in Figure 5(b) are ideal. 


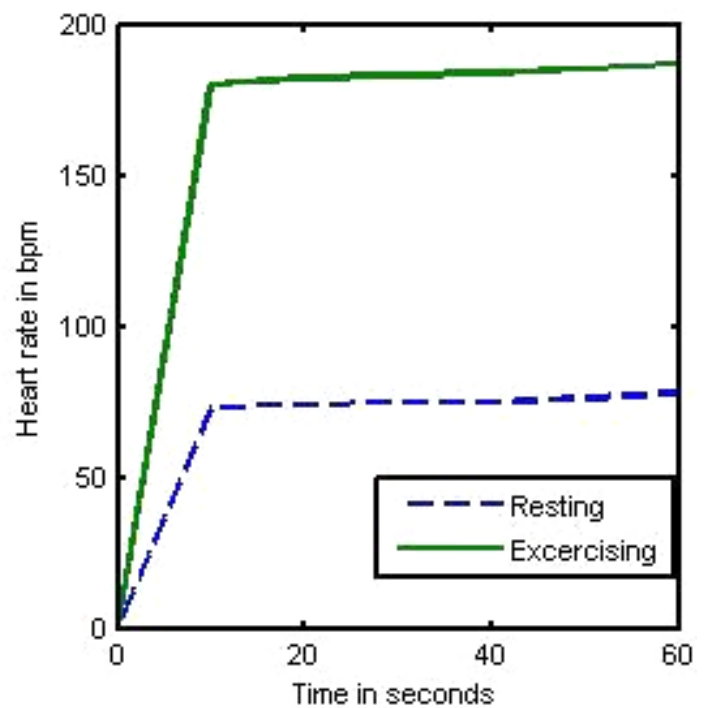

(a)

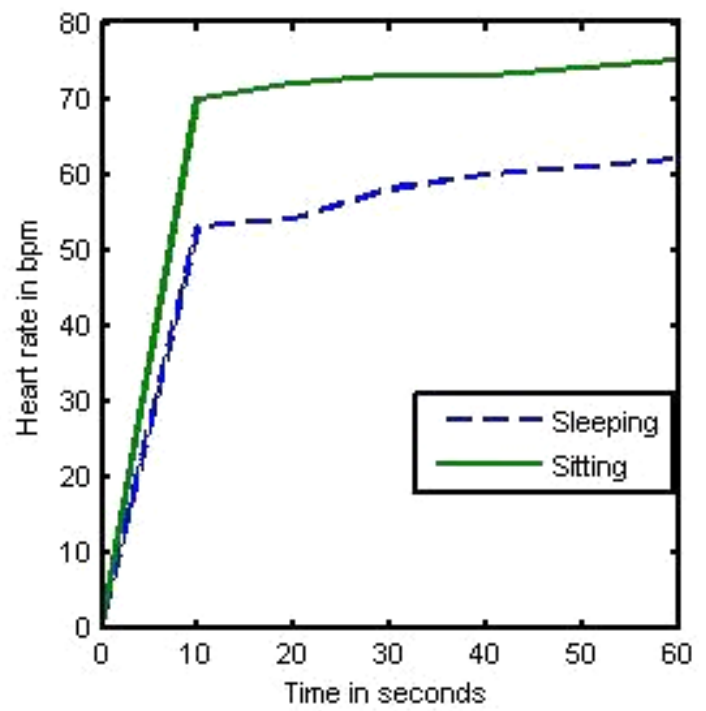

(b)

Figure 5. (a) Resting vs. exercising, and (b) sleeping vs. sitting

\section{4-1-3- Healthy Lifestyle versus Unhealthy Lifestyle}

Figure 6(a) shows a heart rate comparison between the two sample groups. The first group comprises subjects with a healthy lifestyle, while the second does not. This comparison was carried out with 40 of the sampled subjects, excluding COVID-19 patients The result exhibits a clear difference between both heart rate groups. Heart rates in the healthy lifestyle group fell within an acceptable heart rate range, close to ideal, while heart rates in the unhealthy lifestyle group, although within the acceptable range, bordered on the danger zone at $96 \mathrm{bpm}$. It should be noted here that previous studies have found a positive relationship between high sugar consumption and the development of type 2 diabetes. Furthermore, an unhealthy lifestyle can be a direct factor of obesity.

\section{4-1-4- Non-obese versus Obese}

Figure 6(b) shows a heart rate comparison between obese and non-obese subjects, carried out using 40 of the sampled subjects, excluding COVID-19 patients. The results suggest that the non-obese subjects had a regular resting heart rate, whereas the readings for the obese subjects bordered on the danger zone at $94 \mathrm{bpm}$. The reason why obese people tend to have higher resting heart rate is that the greater the amount of bodyfat, resulting in more weight carried, the greater the pressure placed on the heart. Hence, the heart is required to pump harder to dispense blood to other body organs, leading to a faster heartbeat. Therefore, obesity is a critical cause of diabetes. The findings reflect that obesity is a real risk, due to a higher resting heart rate than in non-obese people.

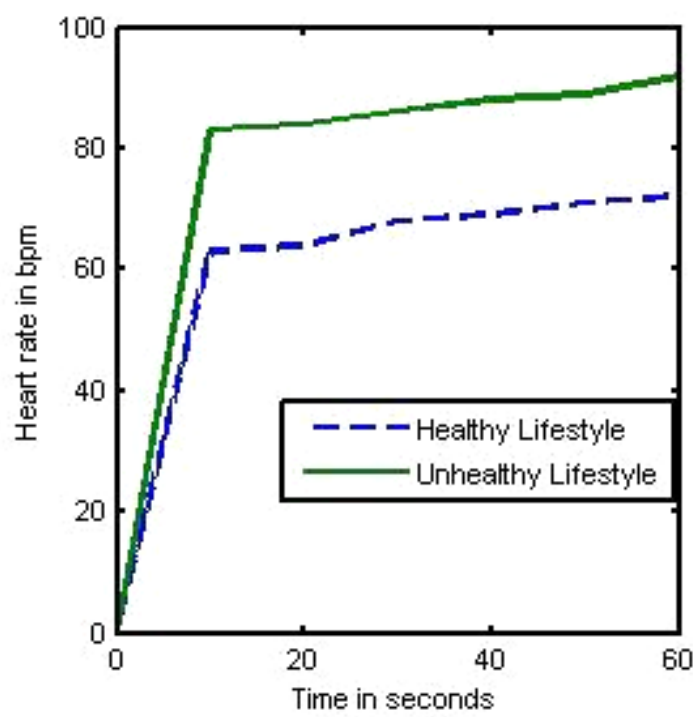

(a)

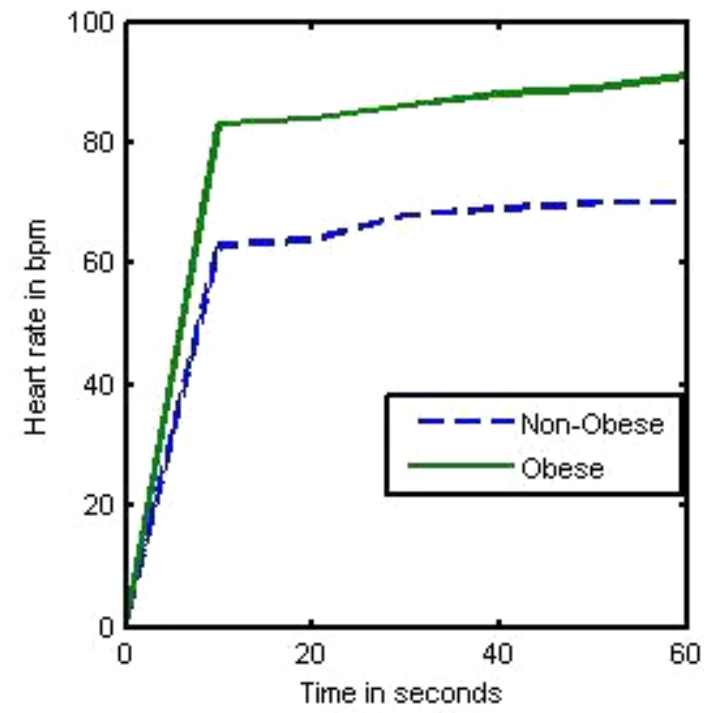

(b)

Figure 6. (a) Healthy lifestyle vs. unhealthy lifestyle, and (b) non-obese vs. obese 


\section{4-1-5- Controlled Diabetic versus Uncontrolled Diabetic}

Figure 7(a) shows a comparison between the heart rate of patients with controlled and uncontrolled diabetes, in a comparison of 20 diabetics in the sample. The result demonstrates that heart rate can be an important predictor of diabetic status, since uncontrolled diabetics tend to have a resting heart rate that is higher than the acceptable rate of $102 \mathrm{bpm}$. This can therefore be used to monitor their daily condition on a continuous basis. Glycaemic status and capillary glucose (level of circulating blood glucose measured by glucometers) are linked to heart rate, but not R-R variability (R-R variability being the physiological phenomenon of fluctuation between heartbeats, calculated by measuring fluctuation in the beat-to-beat interval). In diabetic patients, heart rate recovery was altered during deep breathing and this was linked to lower R-R variability.

\section{4-1-6- COVID-19 Patient versus Uninfected Subject}

Figure 7(b) shows a heart rate comparison between COVID-19 patients and uninfected subjects, conducted among all 50 of the sampled subjects. The result exhibits a clear difference between the groups, as the uninfected subjects displayed a normal heart rate, but the COVID-19 patients had a heart rate of $104 \mathrm{bpm}$, which is dangerous. These results prove what has previously been mentioned regarding the use of heart rate measurement as an early indicator of COVID19. Moreover, uncontrolled diabetes is as dangerous to health as COVID-19 in terms of high heart rate. According to a study conducted using a COVID symptom app, with contributions from four million participants all over the world, an individual's heart rate was found to determine whether an individual had been in contact with the virus. The authors of the above study concluded that COVID-19 could cause a high or irregular heart rate of $100 \mathrm{bpm}$ [29].

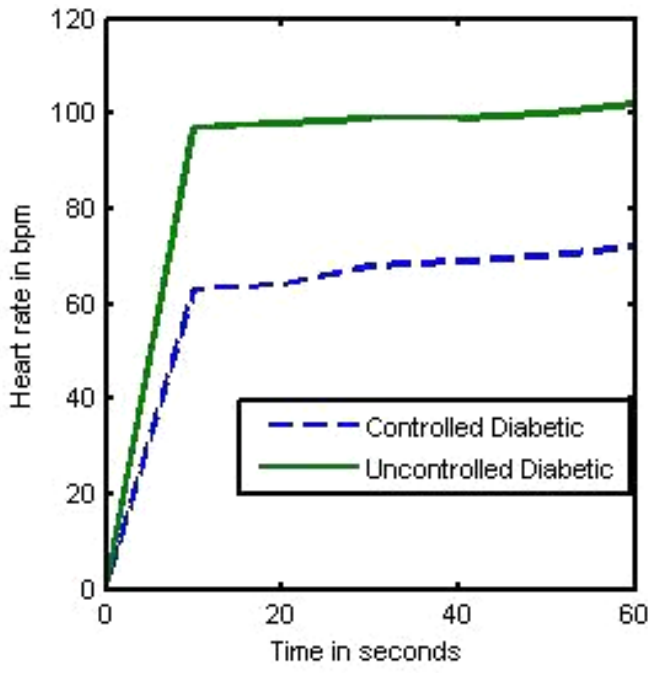

(a)

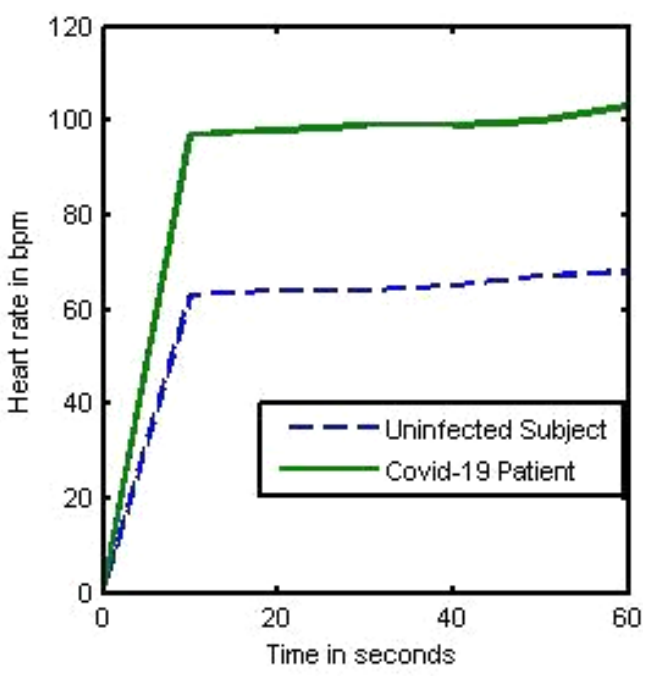

(b)

Figure 7. (a) Controlled diabetic versus uncontrolled diabetic, and (b) COVID-19 patient versus uninfected subject

\section{4-2- Oxygen Saturation (SPO2)}

Oxygen saturation can be an important part of the detection and monitoring of diabetics. One study conducted by the University of Southampton and University College London involved adults being taken up Mount Everest to prove that the restriction of oxygen intake leads to increased insulin resistance. The above study revealed that when the participants reached a higher altitude and were exposed to lower oxygen levels, their insulin resistance developed. By contrast, the opposite effect was observed by researchers from the University of Adelaide when testing the effect of exposing type 2 diabetics to prolonged sessions of hyperbaric oxygen therapy for five weeks. Hyperbaric oxygen therapy involves spending time in a pressurized diving chamber containing 100\% oxygen. The results of the Adelaide experiment appeared to be promising, as they showed $40 \%$ improvement in insulin sensitivity: a result that typically requires $13 \%$ loss of body weight. Oxygen saturation is an important indicator of a person's systemic health. Researchers have also found a relationship between oxygen saturation and COVID-19, whereby 95\%-100\% oxygen saturation implies a healthy individual. However, $90 \%$ oxygen saturation or below is a sign of ill health. Furthermore, $70 \%$ oxygen saturation or below represents confirmation of COVID-19 infection [30]. The results of the present study were compared to those of [13] and commercialised pulse oximeters, revealing very similar results with $99 \%$ accuracy.

\section{4-2-1- Resting versus Exercising}

Figure 8(a) illustrates a comparison between oxygen saturation levels in subjects at rest and engaging in exercise. This comparison was carried out with 30 of the sampled subjects, excluding subjects with COVID-19, uncontrolled 
diabetes, or other medical conditions. The results show that both groups were healthy subjects with $100 \%$ oxygen saturation.

\section{4-2-2- Sleeping versus Sitting}

Figure 8(b) illustrates a comparison between oxygen saturation levels in sleeping or seated subjects. The comparison was carried out with 40 of the sampled subjects, excluding COVID-19 patients. The results show that both groups were healthy, with $100 \%$ oxygen saturation.

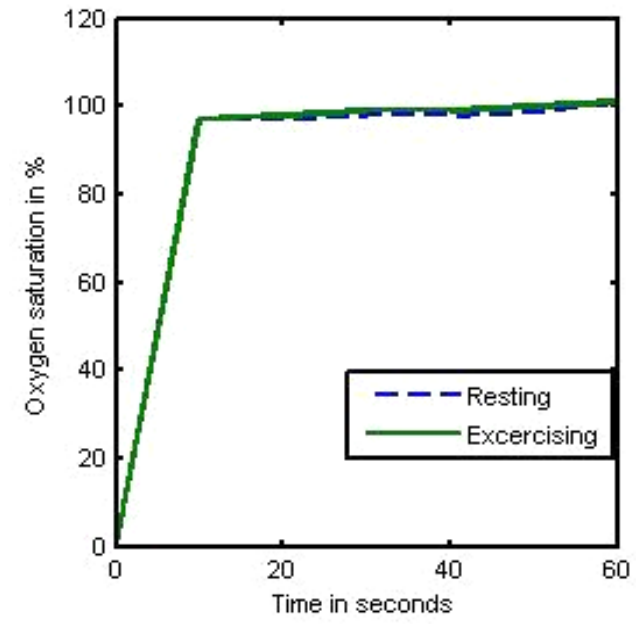

(a)

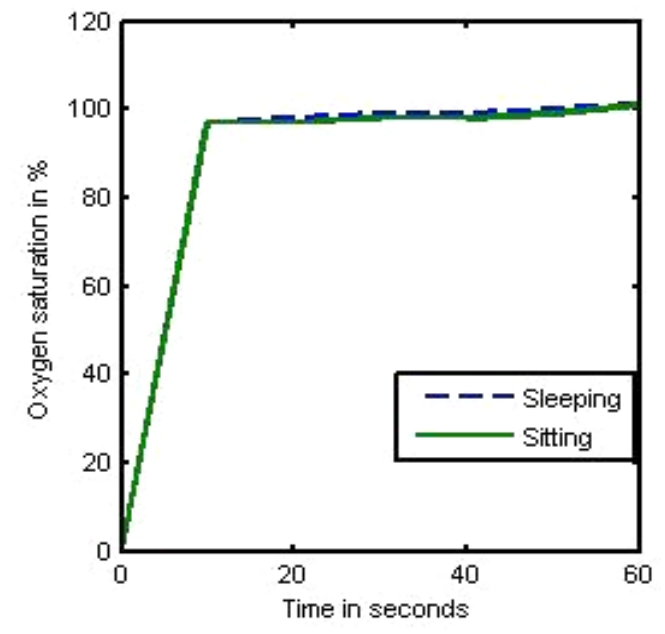

(b)

Figure 8. (a) Resting versus exercising, and (b) sleeping versus sitting

\section{4-2-3- Healthy versus Unhealthy Lifestyle}

Figure 9(a) presents a comparison between oxygen saturation levels among two groups of the sampled subjects: one comprising participants with a healthy lifestyle, and the other made up of participants with an unhealthy lifestyle. The comparison was carried out with 40 of the sampled subjects, excluding COVID-19 patients. The results show that an unhealthy lifestyle is potentially dangerous, with oxygen saturation dropping to the bottom of the acceptable range, bordering on the danger zone at $96 \%$. In contrast, adopting a healthy lifestyle that includes healthy diet and regular exercise can help balance and regulate oxygen saturation levels.

\section{4-2-4- Non-obese versus Obese}

Figure 9(b) illustrates a comparison between oxygen saturation levels in non-obese and obese subjects. This comparison was carried out with 40 of the sampled subjects, excluding COVID-19 patients. The results reflect the same concerns as heart rate for the obese subjects. The oxygen saturation in obese subjects, despite falling within the acceptable range, hovered near the danger zone at $95 \%$. Oxygen saturation decreases in obese subjects with no cardiopulmonary disease, as their body mass index (BMI) increases. The above-mentioned effect may be associated with the energy recovery ventilation (ERV) of an obesity-related reduction in oxygen saturation.

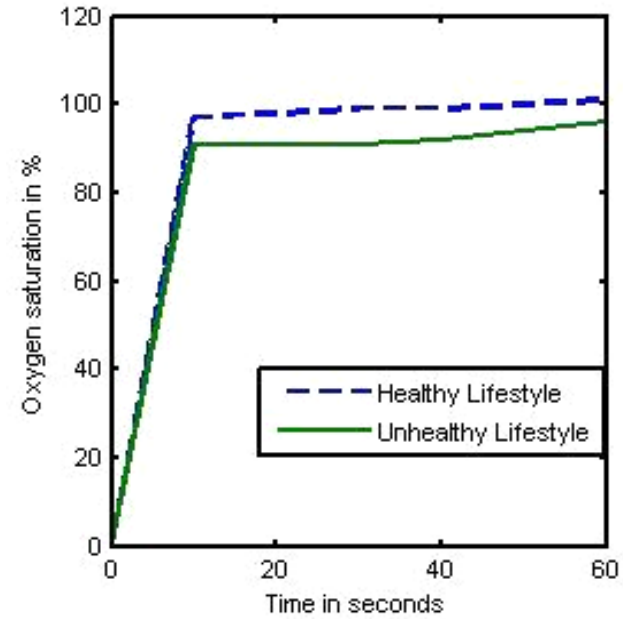

(a)

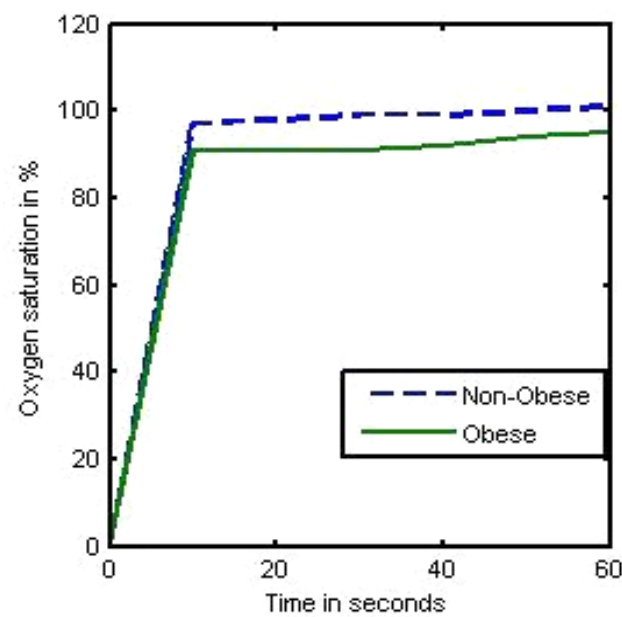

(b)

Figure 9. (a) Healthy lifestyle versus unhealthy lifestyle, and (b) non-obese versus obese 


\section{4-2-5- Controlled versus Uncontrolled Diabetics}

Figure 10(a) shows the oxygen saturation of two groups of subjects with diabetes: one group comprising subjects with controlled diabetes, and the other made up of subjects with uncontrolled diabetes. The results clearly display the risk associated with uncontrolled diabetes, as percentage oxygen saturation fell into the danger zone at $94 \%$. The relationship between oxygen saturation and glucose levels is inverted, meaning that as oxygen saturation decreases, glucose concentration increases in a linear manner. Furthermore, type 1 diabetics tend to have lower oxygen saturation than other diabetic types, measured using pulse oximetry. New research suggests that lower oxygen supply at cellular level can be an early cause and trigger of type 2 diabetes.

\section{4-2-6- COVID-19 Patient versus Uninfected Subject}

Figure 10(b) presents a comparison between oxygen saturation levels in COVID-19 patients and uninfected subjects. This comparison was carried out with all 50 of the sampled subjects. The results show that the COVID-19 subjects had low oxygen saturation levels, with an average of 90-92\%. Previous results have revealed that uncontrolled diabetes is as dangerous to human health as COVID-19 in terms of low oxygen saturation. COVID-19 subjects tend to have low oxygen saturation, even when they are feeling well. As stated earlier, low oxygen saturation can be an early warning and indication that the person requires medical attention/care. However, using the pulse oximeter, the COVID-19 subjects were able to monitor their readings and gain a better understanding of their situation.

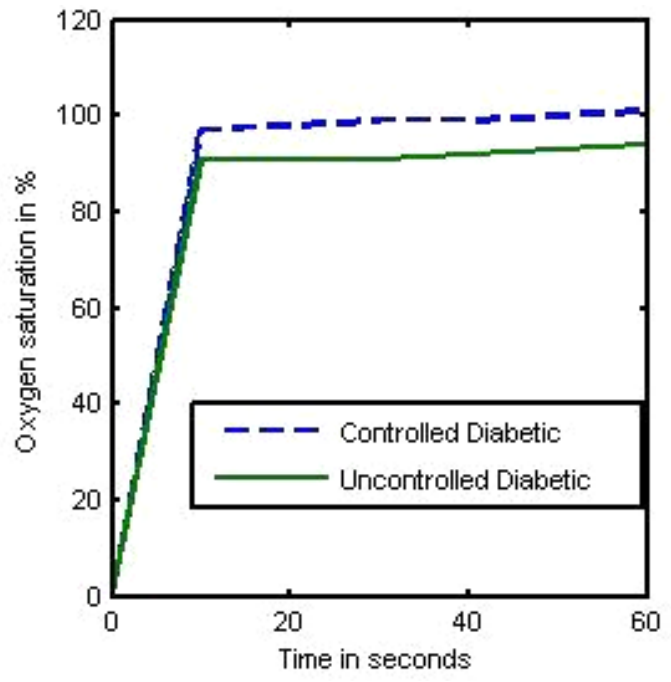

(a)

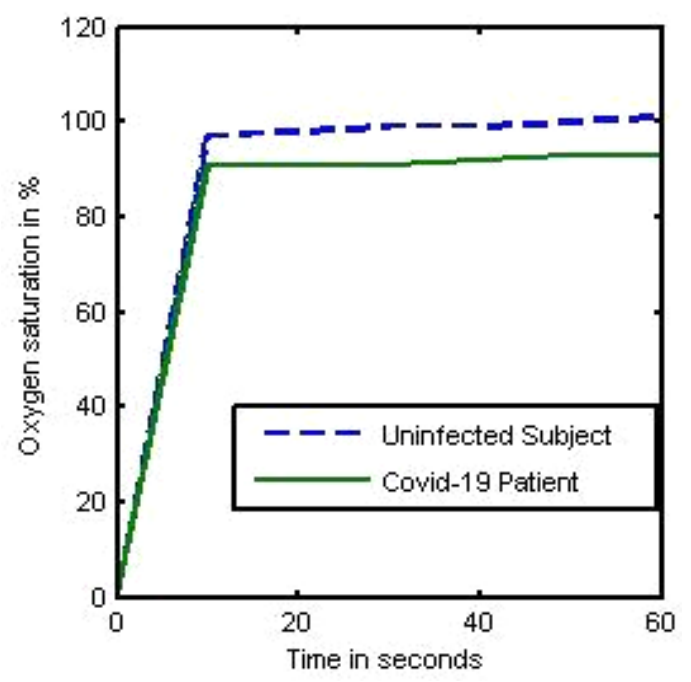

(b)

Figure 10. (a) Controlled diabetic versus uncontrolled diabetic, and (b) COVID-19 patient versus uninfected subject

\section{4-3-Temperature Result}

The average body temperature for the sample of 50 subjects was $37.7^{\circ} \mathrm{C}$. Body temperature was normal among most of the subjects who were uninfected by COVID-19, with their body temperature ranging from $36.4^{\circ} \mathrm{C}$ to $37.3^{\circ} \mathrm{C}$. However, the COVID-19 patients had higher body temperature as a result of the disease, ranging from $37.9^{\circ} \mathrm{C}$ to $38.7^{\circ} \mathrm{C}$. All results were compared to those obtained using a thermometer in commercial use (Medisana Infrared Thermometer), as presented in Table 1.

Table 1. Comparison between the current research results and findings from previous work and other commercial pulse oximeters and thermometers

\begin{tabular}{|c|c|c|c|c|c|c|}
\hline $\begin{array}{l}\text { Max30102 (Heart } \\
\text { Rate in BPM) }\end{array}$ & $\begin{array}{l}\text { Max30102 } \\
(\text { SPO2, \%) }\end{array}$ & $\begin{array}{c}\text { Commercial Pulse } \\
\text { Oximeter (Heart Rate } \\
\text { in BPM, SPO2 \%) }\end{array}$ & $\begin{array}{c}\text { Heart Rate in } \\
\text { BPM, Error \% } \\
{[13]}\end{array}$ & $\begin{array}{l}\text { SPO2 in \%, } \\
\text { Error \%[13] }\end{array}$ & $\begin{array}{c}\text { Max30102 } \\
\left(\text { Temperature }^{\circ} \mathrm{C}\right)\end{array}$ & $\begin{array}{c}\text { Commercial } \\
\text { Thermometer } \\
\left(\text { Temperature }^{\circ} \mathrm{C}\right)\end{array}$ \\
\hline 76 & 100.00 & $79,100.0$ & 96,2 & $98.82,1$ & 36.7 & 36.5 \\
\hline 80 & 98.98 & $82,99.5$ & 92,3 & $99.78,1$ & 36.4 & 36.3 \\
\hline 72 & 99.00 & $73,99.0$ & 76,3 & $98.77,1$ & 36.8 & 37.0 \\
\hline 68 & 100.00 & $72,100.0$ & 74,3 & $97.63,1$ & 37.1 & 37.0 \\
\hline 88 & 97.00 & $91,98.0$ & 80,3 & $99.89,0$ & 36.9 & 37.1 \\
\hline 95 & 95.00 & $99,94.5$ & 98,1 & $98.69,0$ & 37.0 & 36.9 \\
\hline 104 & 92.00 & $105,92.0$ & 93,3 & $99.86,0$ & 37.9 & 38.0 \\
\hline
\end{tabular}

NB. The test subjects differed, in order to compare the current system's accuracy with that of commercial devices and previous research work. 


\section{5- Conclusion}

The research objectives addressed in this paper were met by developing an inexpensive but easy to use and noninvasive system, deploying the Max30102 sensor. Realistic results were obtained, indicating a correlation between oxygen saturation, diabetes, heart rate, and COVID-19, using the non-invasive system designed. The efficacy of this system was achieved by reducing the time in which results were obtained, and finding an internal relationship between metabolic physiological conditions and COVID-19. In addition, the existing pulse oximeter was successfully enhanced by introducing a complete system that could generate results that clearly indicated the effects of age, COVID-19, and controlled and uncontrolled diabetes. Furthermore, the system achieved more accurate results than those produced using conventional systems. When analysed and benchmarked, these results indicated an error of $\pm 3 \%$ for heart rate, $\pm 2 \%$ for temperature, and $\pm 1 \%$ for oxygen saturation. The system could be used more if it was linked to a mobile App, which is a project for the current authors to work on in the future.

\section{6- Declarations}

\section{6-1-Author Contributions}

Conceptualization, A.I.; methodology, A.I.; software, A.I.; validation, A.I., N.A.M., B.A.H., G.H.S., and S.K.; formal analysis, A.I.; investigation, A.I.; resources, A.I., N.A.M., B.A.H., G.H.S., and S.K.; data curation, A.I.; writing — original draft preparation, A.I.; writing—reviewing and editing, A.I., N.A.M., B.A.H., G.H.S., and S.K.; visualization, A.I.; supervision, N.A.M., B.A.H., G.H.S., and S.K.; project administration, A.I. All authors have read and agreed to the published version of the manuscript.

\section{6-2-Data Availability Statement}

The data presented in this study are available on request from the corresponding author.

\section{6-3- Funding}

This research was funded by the Ministry of Higher Education Malaysia (MOHE) through the Fundamentals Research Grant Scheme (FRGS) [Ref.: FRGS/1/2018/TK04/UIAM/02/3].

\section{6-4-Acknowledgements}

Thanks be to Allah the Almighty for giving me the knowledge and strength to produce this paper. I would also like to thank my family and friends, without whose support this paper would not have been a success.

\section{6-5- Conflicts of Interest}

The authors declare that there is no conflict of interests regarding the publication of this manuscript. In addition, the ethical issues, including plagiarism, informed consent, misconduct, data fabrication and/or falsification, double publication and/or submission, and redundancies have been completely observed by the authors.

\section{7- References}

[1] Arden, M. A., \& Chilcot, J. (2020). Health psychology and the coronavirus (COVID-19) global pandemic: A call for research. British Journal of Health Psychology, 25(2), 231-232. doi:10.1111/bjhp.12414.

[2] Our World in Data Organization. (2020). Available online: https://ourworldindata.org (accessed on July 2021).

[3] Pal, R., \& Bhansali, A. (2020). COVID-19, diabetes mellitus and ACE2: The conundrum. Diabetes Research and Clinical Practice, 162. doi:10.1016/j.diabres.2020.108132.

[4] Asmat, U., Abad, K., \& Ismail, K. (2016). Diabetes mellitus and oxidative stress-A concise review. Saudi Pharmaceutical Journal, 24(5), 547-553. doi:10.1016/j.jsps.2015.03.013.

[5] Zhang, P., Zhang, X., Brown, J., Vistisen, D., Sicree, R., Shaw, J., \& Nichols, G. (2010). Global healthcare expenditure on diabetes for 2010 and 2030. Diabetes Research and Clinical Practice, 87(3), 293-301. doi:10.1016/j.diabres.2010.01.026.

[6] Pal, R., \& Bhansali, A. (2020). COVID-19, diabetes mellitus and ACE2: The conundrum. Diabetes Research and Clinical Practice, 162. doi:10.1016/j.diabres.2020.108132.

[7] Guo, W., Li, M., Dong, Y., Zhou, H., Zhang, Z., Tian, C., Qin, R., Wang, H., Shen, Y., Du, K., Zhao, L., Fan, H., Luo, S., \& Hu, D. (2020). Diabetes is a risk factor for the progression and prognosis of COVID-19. Diabetes/Metabolism Research and Reviews, 36(7), 3319. doi:10.1002/dmrr.3319.

[8] Forouhi, N. G., \& Wareham, N. J. (2019). Epidemiology of diabetes. Medicine (United Kingdom), 47(1), $22-27$. doi:10.1016/j.mpmed.2018.10.004. 
[9] Papatheodorou, K., Papanas, N., Banach, M., Papazoglou, D., \& Edmonds, M. (2016). Complications of Diabetes 2016. In Journal of Diabetes Research (Vol. 2016). doi:10.1155/2016/6989453.

[10] Elfström, P., Sundström, J., \& Ludvigsson, J. F. (2014). Systematic review with meta-analysis: Associations between coeliac disease and type 1 diabetes. Alimentary Pharmacology and Therapeutics, 40(10), 1123-1132. doi:10.1111/apt.12973.

[11] Harding, J. L., Pavkov, M. E., Magliano, D. J., Shaw, J. E., \& Gregg, E. W. (2019). Global trends in diabetes complications: a review of current evidence. Diabetologia, 62(1), 3-16. doi:10.1007/s00125-018-4711-2.

[12] Benichou, T., Pereira, B., Mermillod, M., Tauveron, I., Pfabigan, D., Maqdasy, S., \& Dutheil, F. (2018). Heart rate variability in type 2 diabetes mellitus: A systematic review and meta-analysis. PLoS ONE, 13(4), 195166. doi:10.1371/journal.pone.0195166.

[13] Haroon, N., \& Tiwana, M. I. (2017). Design and Development of Non-Invasive Prototype to Measure Pulse Rate, Blood Glucose and Oxygen Saturation Level in Arterial Blood. Future Technologies Conference (November 2017), Vancouver, Canada.

[14] Ionescu, M. (2019). Glucometry and pulse oximetry-comparative noninvasive methods for determining blood glucose. 2019 7th E-Health and Bioengineering Conference, EHB 2019, 1-4. doi:10.1109/EHB47216.2019.8970001.

[15] Pai, P. P., De, A., \& Banerjee, S. (2017). Accuracy enhancement for noninvasive glucose estimation using dual-wavelength photoacoustic measurements and kernel-based calibration. IEEE transactions on instrumentation and measurement, 67(1), 126136. doi:10.1109/TIM.2017.2761237.

[16] Pai, P. P., Kumar Sanki, P., De, A., \& Banerjee, S. (2015). NIR photoacoustic spectroscopy for non-invasive glucose measurement. Proceedings of the Annual International Conference of the IEEE Engineering in Medicine and Biology Society, EMBS, 2015-November, 7978-7981. doi:10.1109/EMBC.2015.7320243.

[17] Jahana, T., \& Higa, H. (2020). Non-Invasive Blood Glucose Monitoring Device Using Photoacoustic Spectroscopy. ICIIBMS 2020 - 5th International Conference on Intelligent Informatics and Biomedical Sciences, 85-88. doi:10.1109/ICIIBMS50712.2020.9336413.

[18] Nanayakkara, N. D., Munasingha, S. C., \& Ruwanpathirana, G. P. (2018). Non-invasive blood glucose monitoring using a hybrid technique. MERCon 2018 - 4th International Multidisciplinary Moratuwa Engineering Research Conference, 7-12. doi:10.1109/MERCon.2018.8421885.

[19] Balasubramanian, S., Kashyap, R., Cvn, S. T., \& Anuradha, M. (2020). Hybrid Prediction Model for Type-2 Diabetes with Class Imbalance. Proceedings of the 2020 IEEE International Conference on Machine Learning and Applied Network Technologies, ICMLANT 2020, 1-6. doi:10.1109/ICMLANT50963.2020.9355975.

[20] Gayathri, B., Sruthi, K., \& Menon, K. U. (2017, April). Non-invasive blood glucose monitoring using near infrared spectroscopy. In 2017 International Conference on Communication and Signal Processing (ICCSP) (pp. 1139-1142). IEEE. doi:10.1109/ICCSP.2017.8286555.

[21] Habibullah, M., Oninda, M. A. M., Bahar, A. N., Dinh, A., \& Wahid, K. A. (2019). NIR-Spectroscopic Classification of Blood Glucose Level using Machine Learning Approach. 2019 IEEE Canadian Conference of Electrical and Computer Engineering, CCECE 2019, 1-4. doi:10.1109/CCECE.2019.8861843.

[22] Kasahara, R., Kino, S., Soyama, S., \& Matsuura, Y. (2018). Noninvasive glucose monitoring using mid-infrared absorption spectroscopy based on a few wavenumbers. Biomedical Optics Express, 9(1), 289. doi:10.1364/boe.9.000289.

[23] Chen, Y., Liu, J., Pan, Z., \& Shimamoto, S. (2020). Non-invasive Blood Glucose Measurement Based on mid-Infrared Spectroscopy. 2020 IEEE 17th Annual Consumer Communications and Networking Conference, CCNC 2020, 1-5. doi:10.1109/CCNC46108.2020.9045160.

[24] Li, X., Huang, X., Mo, J., Wang, H., Huang, Q., Yang, C., Zhang, T., Chen, H. J., Hang, T., Liu, F., Jiang, L., Wu, Q., Li, H., Hu, N., \& Xie, X. (2021). A Fully Integrated Closed-Loop System Based on Mesoporous Microneedles-Iontophoresis for Diabetes Treatment. Advanced Science, 8(16), 2100827. doi:10.1002/advs.202100827.

[25] Yang, J., Li, Y., Ye, R., Zheng, Y., Li, X., Chen, Y., Xie, X., \& Jiang, L. (2020). Smartphone-powered iontophoresis-microneedle array patch for controlled transdermal delivery. Microsystems and Nanoengineering, 6(1), 1-14. doi:10.1038/s41378-02000224-z.

[26] Bolla, A. S., \& Priefer, R. (2020). Blood glucose monitoring- an overview of current and future non-invasive devices. Diabetes and Metabolic Syndrome: Clinical Research and Reviews, 14(5), 739-751. doi:10.1016/j.dsx.2020.05.016.

[27] Swaroop, A., Bagchi, M., Preuss, H. G., \& Bagchi, D. (2018). Safety and Antidiabetic Efficacy of a Novel Trigonella foenumgraecum Seed Extract. Nutritional and Therapeutic Interventions for Diabetes and Metabolic Syndrome, 357-364. doi:10.1016/b978-0-12-812019-4.00028-3. 
[28] Picard, M., Tauveron, I., Magdasy, S., Benichou, T., Bagheri, R., Ugbolue, U. C., Naveli, V., \& Dutheil, F. (2021). Effect of exercise training on heart rate variability in type 2 diabetes mellitus patients: A systematic review and meta-analysis. PLoS ONE, 16(5 May 2021), 251863. doi:10.1371/journal.pone.0251863.

[29] Sudre, C. H., Lee, K. A., Lochlainn, M. N., Varsavsky, T., Murray, B., Graham, M. S., Menni, C., Modat, M., Bowyer, R. C. E., Nguyen, L. H., Drew, D. A., Joshi, A. D., Ma, W., Guo, C. G., Lo, C. H., Ganesh, S., Buwe, A., Pujol, J. C., Du Cadet, J. L., ... Ourselin, S. (2021). Symptom clusters in COVID-19: A potential clinical prediction tool from the COVID symptom study app. Science Advances, 7(12), 4177. doi:10.1126/sciadv.abd4177.

[30] Riddell, M. C., Pooni, R., Fontana, F. Y., \& Scott, S. N. (2020). Diabetes Technology and Exercise. Endocrinology and Metabolism Clinics of North America, 49(1), 109-125. doi:10.1016/j.ecl.2019.10.011. 